\title{
Performance Analysis of VBLAST Based MIMO OFDM System in Vehicular Channel
}

\author{
Samarendra Nath Sur, Soumyasree Bera, Arun Kumar Singh' Rabindranath Bera \\ Department of Electronics and Communication Engineering, Sikkim Manipal Institute of Technology, Sikkim, India \\ B.Maji \\ Department of Electronics and Communication Engineering, National Institute of Technology, Durgapur, West Bengal, \\ India
}

\begin{abstract}
Vehicular communication is emerging as an important ingredient for successful implement of Intelligent Transportation Systems(ITS). But the development of suitable communications systems plays an important role in mobility condition. The desired system should support dynamic wireless exchange of data between nearby vehicles or road side infrastructure. In this regards multiple input and multiple output (MIMO) orthogonal frequency domain multiplexing (OFDM) system is possibly the best solution. This paper deals with the performance analysis of the MIMO OFDM system in Nakagami-m channel with Doppler shift and also partial hardware implementation of baseband signal processing.
\end{abstract}

Index Terms - MIMO, Doppler, OFDM, Nakagami-M Channel, Probability Density Function (PDF)

\section{INTRODUCTION}

ITS consist of several combinations of communication, computer and control technology developed [1] and its main goal is to improve system performance, transport safety, efficiency, productivity, and level of service, environmental impacts, energy consumption, and mobility [2]. The functionality of the ITS is stretch over vast transportation infrastructure of highways, streets, bridges, tunnels, railways, port and airport infrastructure, as well as to a growing number of vehicles, including cars, buses, trucks and trains [1,3].

Lots of initiative have been taken to introduce ITS. Large scale national ITS projects began in Europe, The US and Japan in the mid-1980s. The EU' (European Union) Safety initiative [4], announced in November 2002, aims to halve the number of annual traffic accident fatalities in the EU by 2010 and aim for "zero traffic fatalities" by 2020. The United States' ITS Ten-Year Plan [5], announced in January 2002, talks about achieving a $15 \%$ reduction in the number of annual traffic fatalities by 2011. Europe takes a prime role in regional and international ITS standards activities to advance its ITS technology in the world market. The European Committee for Standardization (CEN) put focused on ITS standards issues. Many ITS standards items are developed in parallel by ISO/TC204 and CEN/TC278 [6]. Japan has been very successful in translating its strengths in electronics technology into successful ITS.
Various forms of wireless communications technologies have been proposed for intelligent transportation systems. Such as Short- range communications using IEEE 802.11 protocols, and Dedicated Short Range Communications (DSRC) standard. From the wireless LAN family, 802.11p/WAVE (Wireless Access for a Vehicular Environments) is developed to provide support in the $5.9 \mathrm{GHz}$ band which has been allocated for ITS in The USA and is under deliberation in Europe and Australia; and a $5.8 \mathrm{GHz}$ allocation in Japan [7]. The aim of CALM is to provide wide area communications to support ITS applications that work equally well on a variety of different network platforms, including Second Generation (2G) mobile (e.g. GSM/GPRS), 3G (IMT-2000 e.g., W-CDMA/CDMA 1x EV-DO) 4G (IMT-Advanced), as well as satellite, microwave, millimetre wave, infrared, WiMAX and WiFi [7]. Recently, two wireless communication schemes, which can be used efficiently to combat with multi-path fading, are extensively investigated around the world. One is MIMO technology and other is the OFDM technology. As an outcome, a combined system, MIMOOFDM, can be used for fourth-generation (4G) mobile cellular wireless system.

From the point of view of the vehicular communication system, mobility may be the challenge or major problems for the next generation communication. In this context $4 \mathrm{G}$ LTE is now operational at different part of the world with $4 \times 4$ antenna configuration. Later LTE-A research works are initiated all over the world to address high data rate with high antenna configuration (max. 8x8) under full vehicular mobility condition and is optimized for low speeds like 0-15 kmph but it supports also speeds upto $350 \mathrm{kmph}$.

This paper addresses the combined VBLAST-MIMOOFDM technique with enhanced performance under high mobility condition.

The paper is organized as follows: The overview of VBLAST MIMO and Nakagami-m channel is discussed in Section II. ICI analysis and BER calculation are presented in Section III and IV respectively. Simulation results and hardware implementation are presented in Section $\mathrm{V}$ and this paper is summarized in Section VI. 


\section{OVERVIEW ON V-BLAST-MIMO SYSTEM AND NAKAGAMI CHANNEL}

\section{A. VBLAST MIMO OFDM System Model}

We consider a V-BLAST MIMO OFDM system with multiple transmitter and receiver antennas with perfect channel state information (CSI) at the receiver. Figure below shows a basic block diagram of a VBLAST MIMO OFDM system.

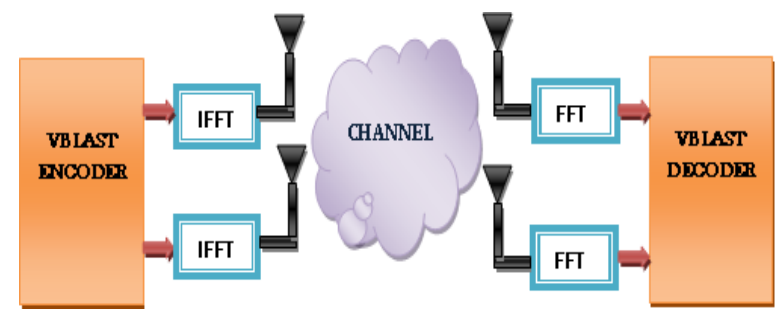

Fig. 1. VBLAST-OFDM system model

At the transmitter incoming bits are mapped using MPSK or MQAM, the layered using VBLAST algorithm. This layered data then feed to the OFDM modulation block. The OFDM signal for each antenna is obtained by applying the inverse Fast Fourier transform (IFFT) on the transmitter side. A cyclic prefix (CP) is inserted in front of the OFDM symbol at the final step of OFDM modulation block. Cyclic prefix is used to reduce the inter symbol interference (ISI). At the receiver side Fast Fourier transform (FFT) is applied after removing the CPs. Then Channel estimation using VBLAST zero forcing (ZF) detection for each OFDM sub-carrier followed by demodulation.

\section{B. Nakagami-m Channel}

The Nakagami-m model serves as a channel model in the highway scenario and is considered as a suitable probabilistic vehicular channel model. However, the transmitter and receiver are moving at high speed in $\mathrm{V} 2 \mathrm{~V}$ environments. This causes different fading statistics depending on the existence of a line-of-sight (LOS). The $\mathrm{m}$ parameter is inversely proportional to distance since the chance of having LOS decreases as distance increases due to turn or intersections on the road.

The PDF of the instantaneous SNR $\gamma$ for the Nakagami $\mathrm{m}$ distribution is $[8,9,10]$ :

$$
\mathrm{P}_{\gamma}(\gamma)=\frac{1}{\Gamma(\mathrm{m})}\left(\frac{\mathrm{m}}{\bar{\gamma}}\right)^{\mathrm{m}} \gamma^{\mathrm{m}-1} \exp \left(\frac{-\mathrm{m} \gamma}{\bar{\gamma}}\right), \quad \gamma \geq 0
$$

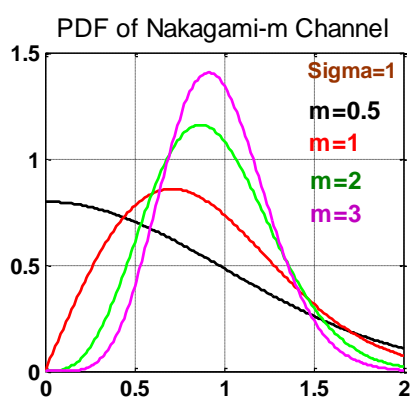

Fig. 2a. PDF of Nakagami channel

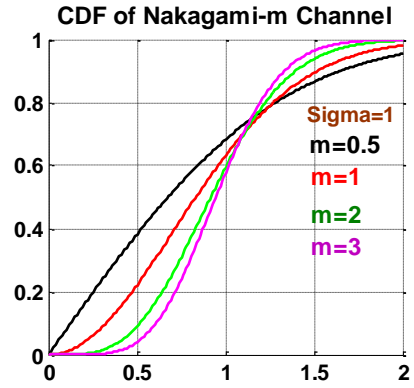

Fig. 2b. CDF of Nakagami channel

Rayleigh fading conditions, i.e. no line-of-sight exists, can be obtained through Nakagami by setting $m=1$. Higher values of $\mathrm{m}$ can be used for approximating Rician distributed channel conditions where a line-of-sight path exists, while for $m<1$, the channel conditions are worse than the Rayleigh distribution.

\section{ICI ANALYSIS}

An OFDM system basically consists of a parallel transmission [11-14]. Each parallel data is mapped with M-ary PSK/ M-QAM digital modulation scheme and, then, those data are modulated by an IFFT on N-parallel subcarriers. After adding cyclic prefix, the parallel data streams are converted to serial one and then the complete OFDM symbol is transmitted over a discrete-time channel. At the receiver, again the serial data stream is converted to parallel one. After the removal of cyclic prefix, the data are retrieved by a FFT and, then, demapped with corresponding scheme to obtain the estimated data.

In an OFDM system the transmitted signal can be represented by

$$
x(n)=\frac{1}{N} \sum_{p=0}^{N-1} x(p) e^{j 2 \pi p n / N}
$$

where $x(n)$ denotes the $n^{\text {th }}$ sample of the OFDM transmitted signal, $X(p)$ denotes the modulated symbol over the $\mathrm{p}^{\text {th }}$ subcarrier and $\mathrm{N}$ is the total number of the subcarriers.

The received signal in time domain after DFT could be written as

$$
Y(p)=\frac{1}{N} \sum_{n=0}^{N-1} y(n) e^{-j 2 \pi p n / N}+G(p)
$$

Where $G(p)$ denotes an additive white Gaussian noise for the $\mathrm{p}^{\text {th }}$ subcarrier.

Assuming the channel frequency offset normalized by the subcarrier frequency spacing denotes as $\varepsilon$ and discrete time domain OFDM signal $\mathrm{y}(\mathrm{n})$ is multiplied by the factor $e^{j 2 \pi n \varepsilon / N}$ in (3) to resent the frequency offset.

As in [11], equation (3) can be written as

$$
Y(p)=S(0) H(p) X(p)+\sum_{1=0, l \neq p}^{N-1} S(1-p) H(l) X(1)+N(p)
$$


Where $\mathrm{p}=0,1$-----, $\mathrm{N}-1$.

The ICI coefficient between $\mathrm{l}^{\text {ih }}$ and $\mathrm{p}^{\text {ih }}$ sub-carrier, $\mathrm{S}(1-$ p) can be expressed as

$$
\begin{aligned}
& \mathrm{S}(1-\mathrm{p})=\frac{\sin (\pi(1-\mathrm{p}+\varepsilon))}{\mathrm{N} \sin \left(\frac{\pi}{\mathrm{N}}(1-\mathrm{p}+\varepsilon)\right)} \exp \left[\mathrm{j} \pi\left(1-\frac{1}{\mathrm{~N}}\right)(1-\mathrm{p}+\varepsilon)\right] \\
& \text { and } \mathrm{S}(0)=\frac{\operatorname{sinc}(\varepsilon)}{\operatorname{sinc}\left(\frac{\varepsilon}{\mathrm{N}}\right)} \exp \left[\mathrm{j} \pi\left(1-\frac{1}{\mathrm{~N}}\right) \varepsilon\right]
\end{aligned}
$$

Here, $S$ (0) produces the phase rotation to the modulated symbol for $\mathrm{p}^{\text {th }}$ subcarrier.

The first term in equation (4) represent the carrier component and the average power on the $\mathrm{p}^{\text {th }}$ can be written as

$$
\mathrm{E}\left[|\mathrm{C}(\mathrm{p})|^{2}\right]=\mathrm{E}\left[|\mathrm{X}(\mathrm{p}) \mathrm{H}(\mathrm{p}) \mathrm{S}(0)|^{2}\right]
$$

The second term represents the ICI component and the average power of it can be written as

$$
\mathrm{E}\left[\left.\mathrm{I}(\mathrm{p})\right|^{2}\right]=\mathrm{E}\left[\left|\sum_{1=0,1 \neq \mathrm{p}}^{\mathrm{N}-1} \mathrm{~S}(1-\mathrm{p}) \mathrm{H}(\mathrm{l}) \mathrm{X}(\mathrm{l})\right|^{2}\right]
$$

The average SINR for the $\mathrm{p}^{\text {th }}$ subcarrier can be represented as

$$
\operatorname{SINR}(p)=\frac{E\left[|X(p) H(p) S(0)|^{2}\right]}{E\left[\left|\sum_{1=0,1 \neq p}^{N-1} S(1-p) H(1) X(1)\right|^{2}\right]}
$$

When SNR is high, the achievable system BER performance in presence of the frequency offset is governed by SINR.

\section{BER CALCULATION}

\section{A. The Zero-Forcing Receiver for MIMO Systems}

Consider a multi-antenna system with $\mathrm{N}_{\mathrm{t}}$ transmit and $\mathrm{Nr}$ receive antennas, where $\mathrm{N}_{\mathrm{t}} \leq \mathrm{N}_{\mathrm{r}}$. We assume a Nakagami-m flat fading channel and can be denoted by a $\mathrm{N}_{\mathrm{t}} \times \mathrm{N}_{\mathrm{r}}$ matrix $\mathrm{H}$. The received signal vector $\mathrm{r}$ can be represented as $\mathrm{r}=\mathrm{Hs}+\mathrm{n}$, where $\mathrm{N}_{\mathrm{t}}$ symbols $\mathrm{s}$ is transmit data symbol vector, $\mathrm{n} \sim \mathrm{CN}\left(0, \mathrm{~N}_{0} \mathrm{I}_{\mathrm{r}}\right)$ is additive white Gaussian noise (AWGN), and $\mathrm{I}_{\mathrm{N}}$ is an $\mathrm{Nx} \mathrm{N}$ identity matrix. If we assume the CSI is perfect, the zero forcing estimate of the transmitted symbol vector and can be expressed as given $\tilde{r}=\mathrm{W}_{\mathrm{ZF}}(\mathrm{Hs}+\mathrm{n})$, where $\mathrm{W}_{\mathrm{ZF}}=\mathrm{H}^{+}=\left(\mathrm{H}^{\mathrm{H}} \mathrm{H}\right)^{-1} \mathrm{H}^{\mathrm{H}}$ the weight vector associated to the $\mathrm{ZF}$ receiver is.

As in [15], the noise covariance can be represented as

$$
\begin{aligned}
& E\left[\hat{n} \hat{n}^{H}\right]= \\
& {\left[\begin{array}{c}
N_{0}+e^{2} N_{0} \operatorname{tr}\left(H^{H} H\right)^{-1} \\
+\frac{4 N_{0} \pi^{2}\left(f_{D} t\right)^{2}}{3} \operatorname{tr}\left(H^{H} H\right)^{-1}
\end{array}\right] \mathrm{X}\left(H^{H} H\right)^{-1}} \\
& +\frac{4 E s \pi^{2}\left(f_{D} t\right)^{2}}{3} I_{N t}
\end{aligned}
$$

where (.) $\mathrm{H}$ denotes Hermitian transpose, e is the quantity that measure the channel estimation accuracy, $f_{D}$ represents the doppler shift and $\operatorname{tr}($.$) represents the matrix$ trace operation.

The SINR per symbol of each stream can be represented as

$$
\bar{\gamma}=\frac{\mathrm{Es}_{0} / \mathrm{N}_{0}}{\left(\left[1+\mathrm{e}^{2} \mathrm{~N}_{\mathrm{t}} \mathrm{E}_{\mathrm{s}} / \mathrm{N}_{0}\right]+\left(\frac{4 \mathrm{Es} \pi^{2}\left(\mathrm{f}_{\mathrm{D}}\right)^{2}}{3 \mathrm{~N}_{0}}\right)\right)}
$$

\section{B. BER calculation for MPSK modulation.}

The moment generating function (MGF) $[16,17]$ of the SNR in Nakagami-m fading is given by

$$
\begin{aligned}
\varphi_{\gamma}(s) & =\int_{0}^{\infty} \exp (-s \gamma) P_{\gamma}(\gamma) d \gamma \\
& =\left(\frac{m}{m+s \gamma}\right)^{m}, m \geq \frac{1}{2}
\end{aligned}
$$

The conditional- error probability (CEP) for MPSK is given by

$$
P_{s}(\gamma)=\frac{1}{\pi} \int_{0}^{\pi-\pi / M} \exp \left(\frac{-\gamma \sin ^{2}(\pi / M)}{\sin ^{2} \theta}\right) d \theta
$$

The average symbol error rate in Nakagami channel for positive value of fading depth $\mathrm{m}$ is given by

$$
\begin{aligned}
\overline{\mathrm{P}_{\mathrm{S}}} & =\int_{0}^{\infty} \mathrm{P}_{\mathrm{S}}(\gamma) \mathrm{P}_{\gamma}(\gamma) \mathrm{d} \gamma \\
& =\frac{1}{\pi} \mathrm{I}_{\mathrm{S}}\left(0, \pi-\pi / \mathrm{M},\left(\frac{\bar{\gamma}}{\mathrm{m}}\right) \sin ^{2}(\pi / \mathrm{M}), \mathrm{m}\right)
\end{aligned}
$$

As in [16], the generalized form of $I_{S}$ can be expressed as

$$
\begin{aligned}
I_{s}\left(\theta_{L, \theta_{U}} c, d\right) & =\int_{\theta_{L}}^{\theta_{U}}\left[\frac{\sin ^{2} \theta}{c+\sin ^{2} \theta}\right]^{d} d \theta \\
& =
\end{aligned}
$$




$$
\begin{aligned}
& \theta_{\mathrm{U}}-\theta_{\mathrm{L}}-\sqrt{\frac{\mathrm{c}}{\mathrm{c}+1}}\left[\frac{\pi}{2}+\lambda\left(\mathrm{c}, \theta_{\mathrm{U}}\right)\right]_{\mathrm{k}=0}^{\mathrm{d}-1} \frac{1}{[4(\mathrm{c}+1)]^{\mathrm{k}}} \\
& \times\left\{\left(\begin{array}{l}
2 \mathrm{k} \\
\mathrm{k}
\end{array}\right)+\frac{1}{\pi} \sum_{\mathrm{h}=0}^{\mathrm{k}-1}\left(\begin{array}{l}
2 \mathrm{k} \\
\mathrm{h}
\end{array}\right) \frac{\sin \left[2(\mathrm{k}-\mathrm{h}) \lambda\left(\mathrm{c}, \theta_{\mathrm{U}}\right)\right]}{\mathrm{k}-\mathrm{h}}\right\}+ \\
& \sqrt{\frac{\mathrm{c}}{\mathrm{c}+1}\left[\frac{\pi}{2}+\lambda\left(\mathrm{c}, \theta_{\mathrm{L}}\right)\right]_{\mathrm{k}=0}^{\mathrm{d}-1} \frac{1}{[4(\mathrm{c}+1)]^{\mathrm{k}}}} \\
& \times\left\{\left(\begin{array}{l}
2 \mathrm{k} \\
\mathrm{k}
\end{array}\right)+\frac{1}{\pi} \sum_{\mathrm{h}=0}^{\mathrm{k}-1}\left(\begin{array}{l}
2 \mathrm{k} \\
\mathrm{h}
\end{array}\right) \frac{\sin \left[2(\mathrm{k}-\mathrm{h}) \lambda\left(\mathrm{c}, \theta_{\mathrm{L}}\right)\right]}{\mathrm{k}-\mathrm{h}}\right\}
\end{aligned}
$$

Where $\mathrm{d}$ is a positive integer and

$$
\begin{aligned}
& \lambda(\mathrm{q}, \theta)=\tan ^{-1}\left[\sqrt{\left(\frac{\mathrm{q}}{1+\mathrm{q}}\right)} \cot (\pi-\theta)\right] \\
& \mathrm{c}=\left(\frac{\bar{\gamma}}{\mathrm{m}}\right) \sin ^{2}(\pi / \mathrm{M})
\end{aligned}
$$

$\mathrm{L}=$ Total no of diversity branches, $\mathrm{h}=(0,1,2 \ldots \mathrm{L})$ and $\mathrm{k}=\mathrm{k}^{\text {th }}$ signal point with instantaneous SNR $\gamma$

\section{C.BER calculation for MQAM modulation}

The BER of coherent M-QAM with two-dimensional Gray coding over the AWGN channel.

$$
\mathrm{P}_{\mathrm{b}}(\gamma) \cong 0.2 \exp \left(\frac{-3 \gamma}{2(\mathrm{M}-1)}\right)
$$

The average BER in a slow and fat Nakagami-m fading channel may be derived by averaging the error rates for the AWGN channel over the pdf of the SNR in Nakagami-m fading:

$$
\begin{aligned}
& \overline{\mathrm{P}_{\mathrm{b}}(\gamma)}=\int_{-\infty}^{\infty} \mathrm{P}_{\mathrm{b}}(\gamma) \times \mathrm{P}_{\gamma}(\gamma) \mathrm{d} \gamma \\
& =\int_{0}^{\infty} 0.2 \exp \left(\frac{-3 \gamma}{2(M-1)}\right) \times \frac{1}{\Gamma(m)}\left(\frac{m}{\bar{\gamma}}\right)^{m} \gamma^{m-1} \exp \left(\frac{-m \gamma}{\bar{\gamma}}\right) d \gamma
\end{aligned}
$$

Now after some simplification as in [18], the average BER can be calculated as given below

$$
\overline{\mathrm{P}_{\mathrm{b}}(\gamma)}=0.2\left(\frac{2 \mathrm{~m}(\mathrm{M}-1)}{3 \bar{\gamma}+2 \mathrm{~m}(\mathrm{M}-1)}\right)^{\mathrm{m}}
$$

Applying (11) to the BER expression of M-PSK and MQAM for MIMO ZF receiver in equation (15) and (20), we can have the dependence of BER on the Doppler shift.

\section{RESULTS}

\section{A. Simulation results}

Above figure shows the BER performance of the $2 \times 2$ VBLAST OFDM system in Nakagami channel condition. As in figure with the change in $\mathrm{m}$ values the performance of the system gets better. This improvement in the BER performance is due to the basic fading characteristics of the Nakagami channel, as with the increment of the $\mathrm{m}$ value Nakagami fading characteristics changes from one sided Gaussian distribution to Raleigh distribution. As in figure with the change in $m$ values at the high SNR level the system performance improves dramatically with respect to that of in low SNR level.

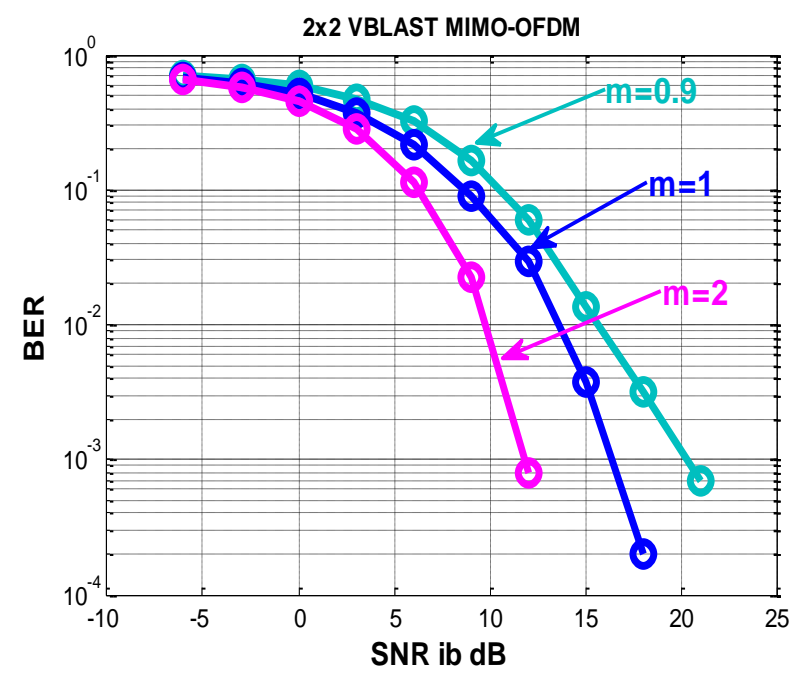

Fig. 3. SNR vs. BER Curves with different $m$ values for Nakagami-m channel.

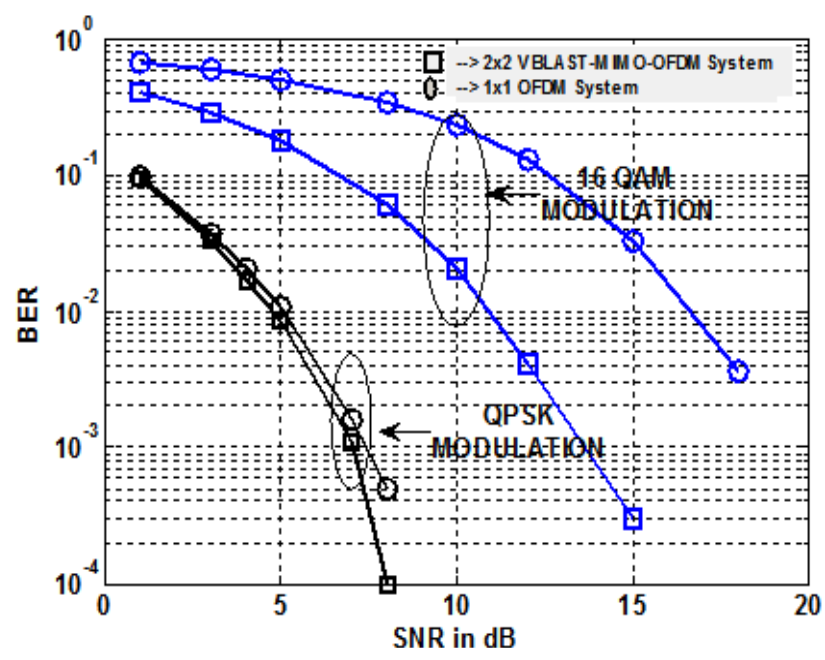

Fig. 4. SNR vs. BER Curves with $200 \mathrm{~Hz}$ Doppler frequency

Figure 4 represent performance analysis of $2 \times 2$ VBLAST MIMO-OFDM system and SISO OFDM system with $200 \mathrm{~Hz}$ Doppler frequency. As in figure, with the lower order modulation (here QPSK) the performance gap between the above said two systems is narrow whereas in case of higher order modulation (here QAM) the performance gap between the above said two systems is noticeable. Therefore one can conclude that a combination of MIMO -OFDM is much more effective in case of higher order modulation and in severe channel condition in comparison to SISO OFDM system.

By comparing above curves, it is clear that VBLASTMIMO-OFDM system provide better performance in comparison to that of VBLAST MIMO system. 


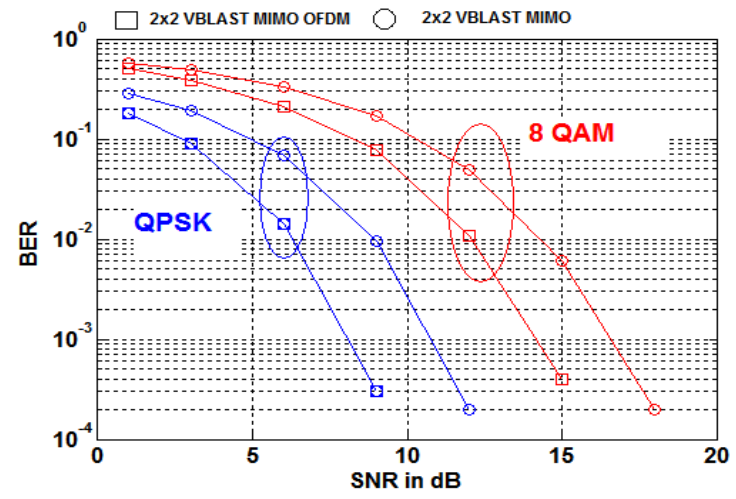

Fig. 5. SNR vs. BER Curves for VBLAST MIMO OFDM and VBLAST MIMO

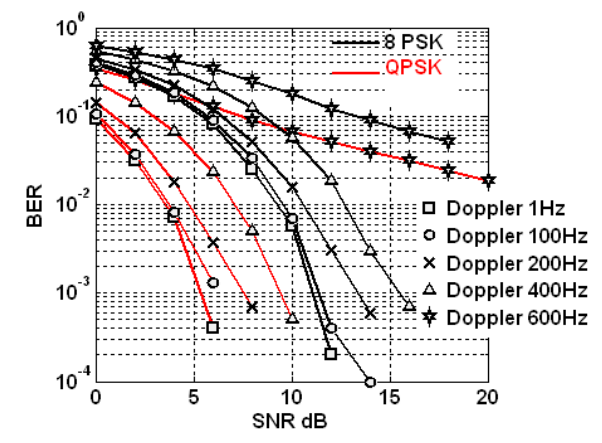

From figures 4 and 6 , one can conclude that VBLAST MIMO OFDM have much more tolerance against the Doppler shift and can support the velocity upto $90 \mathrm{KM} / \mathrm{hr}$ (400 Hz Doppler and in $2.4 \mathrm{GHz}$ band) and useful for vehicular application.

\section{B. Hardware Experimentation:}

Figure 7 shows the basic block diagram which is implemented and realized through arbitrary waveform generator (AWG 5014C) and Vector signal analyzer (VSA 89600). Both the equipments are programmable from compute using MATLAB SIMULINK. The basic data generations, BPSK modulation, 2 x 2 VBLAST encoder, training insertion have been modeled in MATLAB SIMULIK environment. The data corresponding to SIMULINK model is first saved to *.mat and subsequently converted to *.txt file which is then ported to AWG for the realization of the transmitter. Furthermore the multipath channel is modeled and implemented in AWG. The generation of multipath channel is indeed an inherent feature of the AWG. During implementation we have considered $300 \mathrm{MHz}$ as $\mathrm{RF}$ carrier with a bandwidth of $100 \mathrm{MHz}$ of signal.

Fig. 6. SNR vs. BER Curves for $2 \times 2$ VBLAST MIMO OFDM with different Doppler shifts.

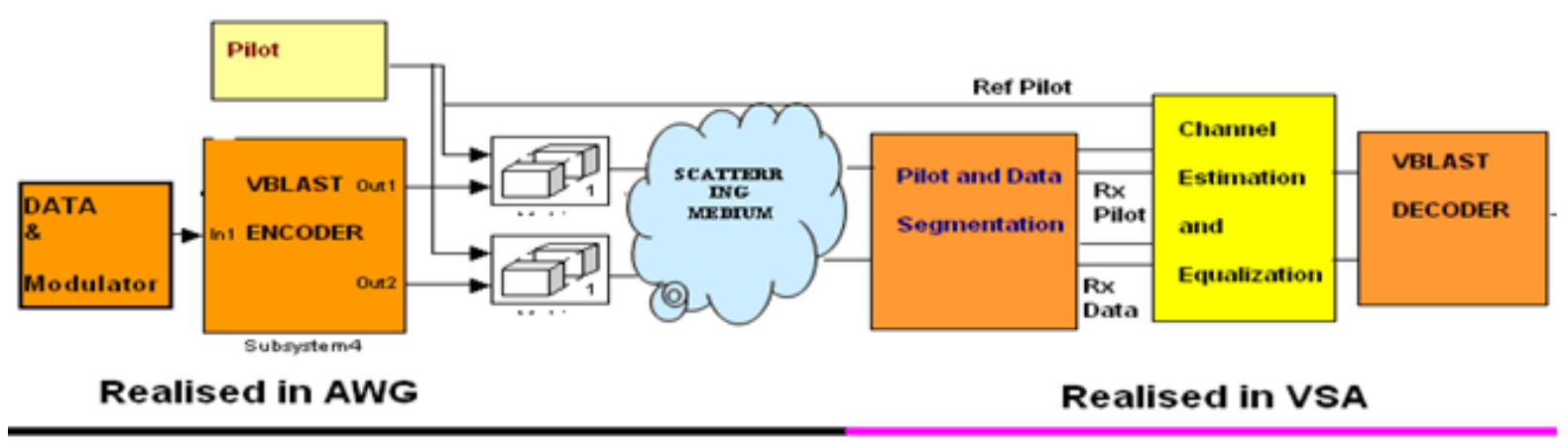

Fig. 7. Basic Block Diagram for Hardware Realization

Agilent VSA is a versatile signal analyzer of a new kind of instruments fully programmable via its own embedded Agilent 89600 VSA software and it is programmable from computer through MATLAB/SIMULINK. In VSA, the $1^{\text {st }}$ job is to segregate the pilot from the data, then channel estimation and equalization. In the last stage $2 \times 2$ VBLAST decoder is implemented.

The total experiment is done in closed loop fashion with AWG and VSA (Figure 8).

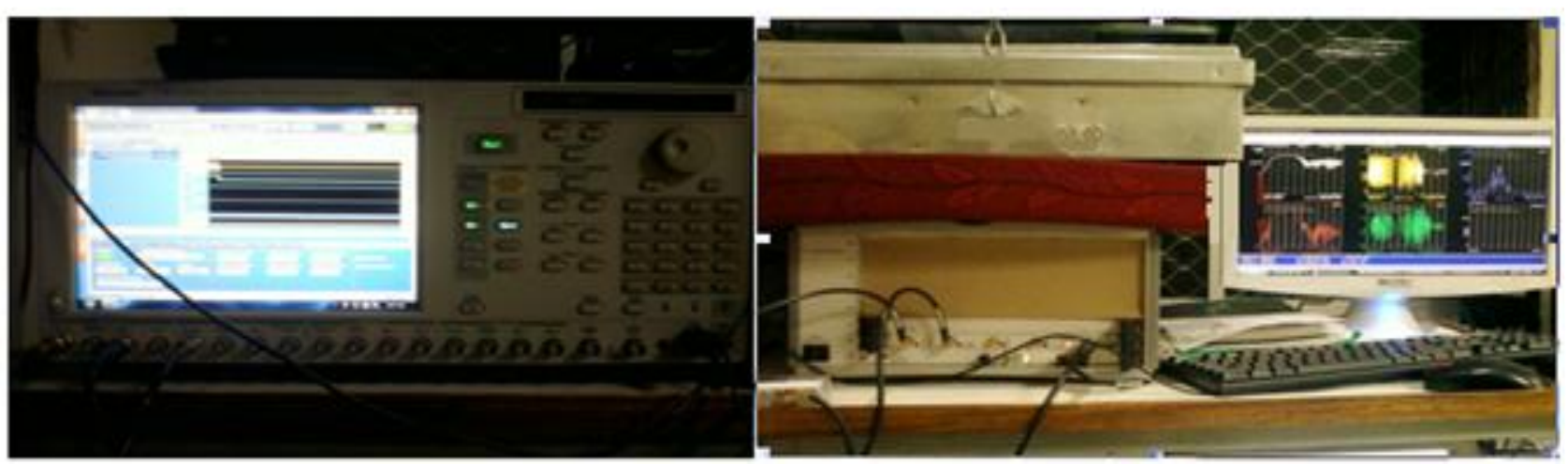

Fig. 8. Closed loop setup consist of AWG and VSA 


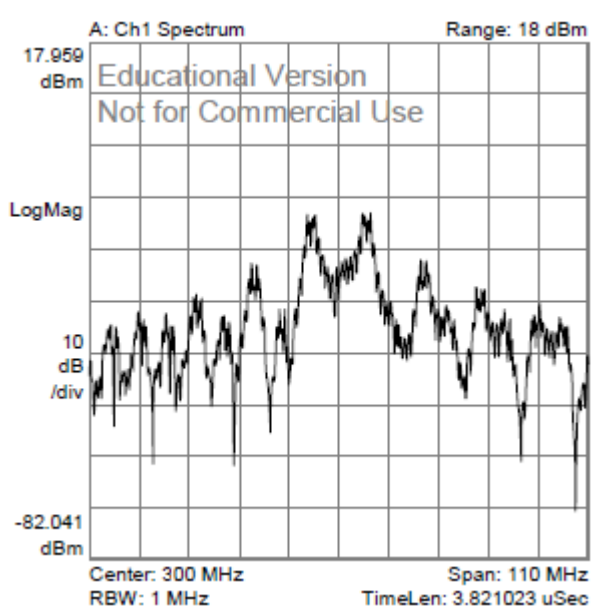

Fig. 9. Signal spectrum at $300 \mathrm{MHz}$ RF frequency.

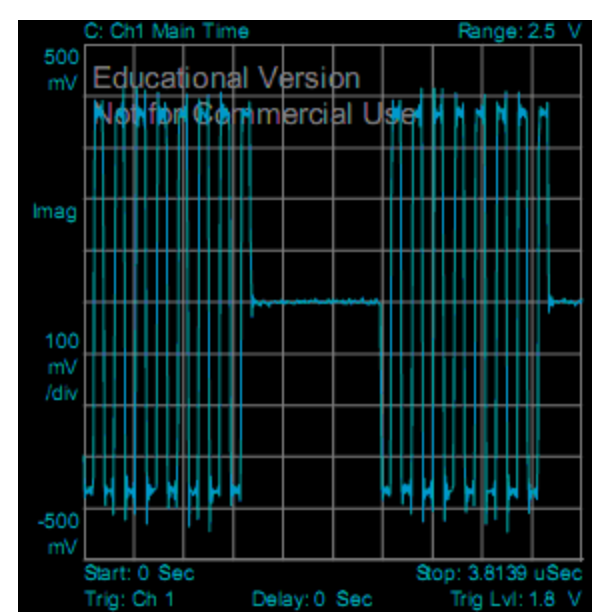

Fig. 10. Information data (Base band).

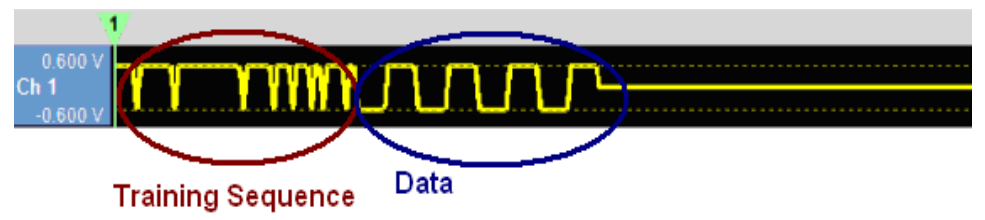

Fig. 11. Baseband Signal at the transmitter side after training symbol insertion for 1st channel (Real part).

As in the figure 7, information data stream is first modulated and the it is VBLAST encoded. Then training data stream is added to both of the coded data. Figure 11 shows the real part of the $1^{\text {st }}$ transmitted data stream for channel 1 .
After pilot insertion the data streams are up-converted to the RF level of $300 \mathrm{MHz}$ and passed through channel. Figure below shows the signal after passing through channel.

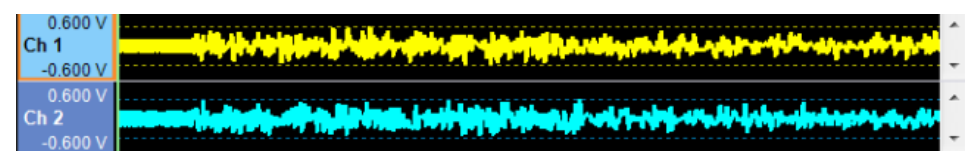

Fig. 12. RF Signal from AWG corrupted by the channel (-3dB SNR)

As in figure 12, because of low the SNR signal quality degraded as the noise floor over shoot the signal level.

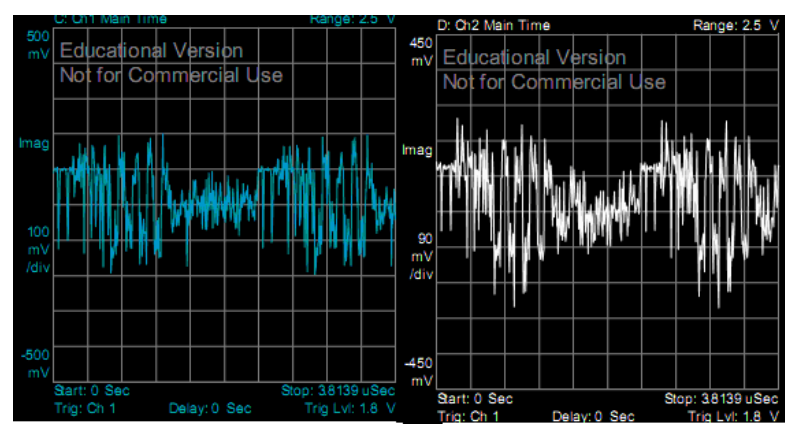

Fig. 13. Base band Received Signal at VSA (with -3dB SNR)

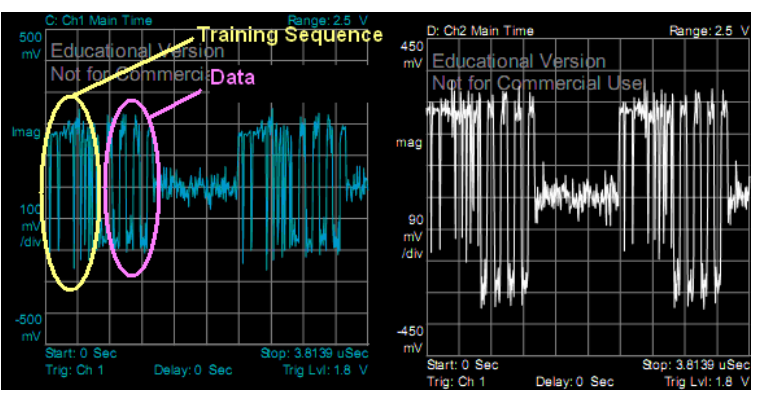

Fig. 14. Base band Received Signal at VSA (with 6 dB SNR)
Figure 13 and 14 represent the received base band signal at the VSA. It is clear from the figures that as the SNR value decrease the signal quality degraded.

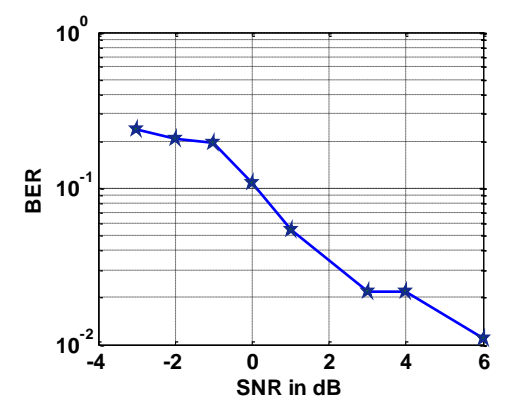

Fig. 15. SNR vs BER curve in closed loop condition.

Above figure shows the BER performance of the above said VBLAST system in closed loop form with AWG and VSA. Figure shows with the increase in SNR value the BER reduces.

\section{CONCLUSION}

Through the simulation results, it is found that $2 \times 2$ VBLAST MIMO OFDM system provide better 
performance in comparison to single antenna OFDM system and conventional 2x2 VBLAT MIMO system. And MIMO-OFDM system provides better resistance against Doppler shift. Therefore VBLAST MIMO OFDM system is one of the best and suitable solutions in high mobility condition

\section{REFERENCES}

[1] http://www.transport-research.info/web/themes/ technology.cfm

[2] Thematic Research Summary: "Intelligent transport systems", Transport Research Knowledge Centre, European commission, DG Energy and Transport, Prepared by Zuzana !itavancová Martin Hájek, Date 02/04/2009.

[3] http://egov.eletsonline.com/2012/08/sustainable-publictransportation-through-ict/

[4] EU. eSafety, "Final Report of the eSafety Working Group on Road Safety", November, pp.7-39, 2002

[5] J. K. Hedrick, et al., "Control Issues in Automated Highway Systems”, IEEE Control Systems, Vol.14, No. 6, pp.21-32, 1994

[6] ITS Technical Note For Developing Countries, Toshiyuki Yokota, Sr . Transport Specialist Transport and Urban Development Department.

[7] 'Intelligent Transport Systems and CLAM' ITU Technology Watch Report 1, October 2007 (http://www.scribd.com/doc/17501030/IntelligentTransport-Systems-and-CALM)

[8] Hyundong Shin, and JaeHong Lee, "On the Error Probability of Binary and M-ary Signals in Nakagami-m Fading Channels" IEEE TRANSACTIONS ON COMMUNICATIONS, VOL.52, NO.4, APRIL2004.

[9] Li Tang, Zhu Hongbo,"Analysis and Simulation of nakagami fading channel with Matlab,"Asia-Pacific conference on environmental electromagnetic CEEM'2003, China,pp. 490-494.

[10] M. Nakagami, "The m-distribution, a general formula of intensity distribution of rapid fading," in Statistical Methods in Radio Wave Propagation, W. G. Hoffman, Ed, Oxford, England: Pergamum, 1960.

[11] Himal A. Suraweera and Jean Armstrong, "Performance of V-BLAST MIMO-OFDM Systems with Carrier Frequency Offset" Au sCTW 2005, 2005, pp-73-78

[12] J. Armstrong, P. M. Grant and G. Povey, "Polynomial cancellation coding of OFDM to reduce the intercarrier interference due to Doppler spread," in Proc. IEEE GLOBECO M 98 , Sydney, Australia, Nov. 1998 , pp. 2771-2776.

[13] Srabani Mohapatra and Susmita Das, "Performance Enhancement of OFDM System with ICI Reduction Technique", Proceedings of the World Congress on Engineering 2009, Vol 1, WCE 2009, July 1-3, 2009.

[14] Samarendra Nath Sur and Rabindranath Bera, "Doppler Shift Impact On The MIMO OFDM System In Vehicular Channel Condition" IJITCS, VOL.4, NO.8, July, 2012, pp- 57-62.

[15] Sang Goo Kim, Dongweon Yoon, Zhengyuan $\mathrm{Xu}$, and Sang Kyu Park, "Performance Analysis of the MIMO Zero-Forcing Receiver over Continuous Flat Fading Channel”, ICIS, 23-25 June 2010

[16] A. Annamalai, and C. Tellambura, "Error Rates for Nakagami-m Fading Multi-channel Reception of Binary and M-ary Signals", IEEE TRANSACTIONS ON COMMUNICATIONS, VOL. 49, NO.1, JANUARY 2001.
[17] M.K. Simon and M.-S. Alouini, "Digital Communication Over Fading Channels :A Unified Approach to Performance Analysis". NewYork: Wiley,2000.

[18] Tahmid Quazi and HongJun Xu1, "Performance analysis of adaptve M-QAM over a fat-fading Nakagami-m channel", S Afr J Sci. 2011;107(1/2), Art. \#122, 7 pages. DOI: $10.4102 /$ sajs.v107i1/2.122

\section{Authors' Profiles}

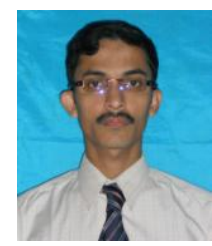

Mr. Samarendra Nath Sur: Born in 1984 at Hooghly, West Bengal, INDIA He received his M.Sc. (Electronics Science) from Jadavpur University in the year 2007 and M.Tech from Sikkim Manipal University in 2012. Currently working as an Assistant Professor in Electronics \& Communication Engineering Department of Sikkim Manipal Institute of Technology, India. Broadband Wireless Communication Advanced Digital Signal Processing and Remote Sensing are the area of specializations.

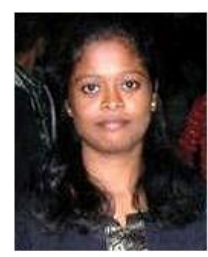

Ms. Soumyasree Bera: Born in 1988 at Kolaghat, West Bengal, INDIA. She received her B. Tech from Sikkim Manipal Institute of Technology, Sikkim Manipal University, in the year 2011. Currently she is working as Faculty in the Department of Electronics \& Communication Engineering, in same college. Her area of Interest includes Remote Sensing, Mobile Communication, Advanced Digital Signal Processing.

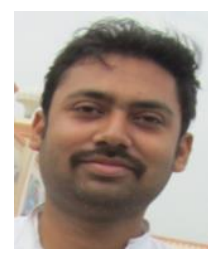

Mr. Arun Kumar Singh: Born in 1988 in Bihar, India, received his B.tech, M.tech Degree in Electronics and Communication Engineering dept. from Sikkim Manipal Institute of Technology. His area of interest is remote sensing, mobile communication and satellite communication.

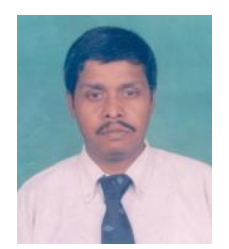

Prof. (Dr.) Rabindranath Bera: Born in 1958 at Kolaghat, West Bengal, INDIA. Received his B. Tech, M. Tech \& Ph.D degree from the Institute of Radiophysics \& Electronics, The University of Calcutta, in the year 1982, 1985 \& 1997 respectively. Currently working as Professor and Head of the Department, Electronics \& Communication Engineering. Sikkim Manipal University, Sikkim. . His major research Interests in the area of Digital Radar, RCS Imaging, Wireless 4G Communication, Radiometric remote sensing. He has published large number of papers in different national and international Conference and journals.

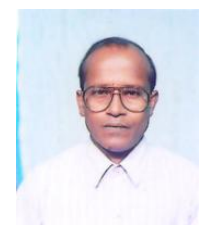

Prof. (Dr.) Bansibadan Maji, He currently working as a senior Professor of Electronics \& Communication Engineering Department in NIT, Durgapur, West Bengal, India. Presently he is Head of The Department of ECE at NIT. His main research area on Microwave, Antenna, VLSI Design and Low power Device and Circuits. He has more than 56 publications in different International and National Journals and Conference Proceedings. 